\title{
Convection in superposed fluid and porous layers
}

\author{
By FALIN CHEN ${ }^{1}$ AND C. F. CHEN ${ }^{2}$ \\ ${ }^{1}$ Institute of Applied Mechanics, National Taiwan University, Taipei, Taiwan 10764, ROC \\ ${ }^{2}$ Department of Aerospace and Mechanical Engineering, The University of Arizona, \\ Tucson, AZ 85721, USA
}

(Received 20 October 1988 and in revised form 6 June 1991)

A nonlinear computational investigation of thermal convection due to heating from below in a porous layer underlying a fluid layer has been carried out. The motion of the fluid in the porous layer is governed by Darcy's equation with the Brinkman terms for viscous effects and the Forchheimer term for inertial effects included. The motion in the fluid layer is governed by the Navier-Stokes equation. The flow is assumed to be two-dimensional and periodic in the horizontal direction, with a wavelength equal to the critical value at onset as predicted by the linear stability theory. The numerical scheme used is a combined Galerkin and finite-difference method, and appropriate boundary conditions are applied at the interface. Results have been obtained for depth ratios $\hat{d}=0,0.1,0.2,0.5$ and 1.0 , where $\hat{d}$ is the ratio of the thickness of the fluid layer to that of the porous layer. For $\hat{d}=0.1$, up to $R_{\mathrm{m}}$ (Rayleigh number of the porous layer) equal to 20 times the critical $R_{\mathrm{m}_{\mathrm{c}}}$, the convection is dominated by the porous layer, similar to the situation at onset, even though the Rayleigh number for the fluid layer is well into the supercritical regime. The Nusselt number for $\hat{d}$ less than the critical value (0.13 in the present case) increases sharply with $R_{\mathrm{m}}$, whereas at larger $\hat{d}$, the increase is very moderate. Heat transfer rates predicted by the numerical scheme for $\hat{d}=0.1$ and 0.2 show good agreement with the experimental results of Chen \& Chen (1989).

\section{Introduction}

In Chen \& Chen (1988) we have shown that when a fluid layer superposed on a porous layer is heated from below, the linear stability theory predicts that there is a critical depth ratio, the ratio of the thickness of the fluid layer to that of the porous layer. For depth ratios less than the critical value, onset of thermal convection is in the form of large convection cells in the porous layer with wavelength comparable to the depth of the porous layer. For depth ratios larger than the critical value, the onset of thermal convection is confined within the fluid layer. Thus, the marginal stability curve is bimodal, and at the critical depth ratio there is a dramatic increase in the critical wavenumber by one order of magnitude. For a porous layer consisting of $3 \mathrm{~mm}$ diameter glass beads saturated with water, the critical depth ratio is 0.13 . The theoretical prediction has been verified by experimental results (Chen \& Chen 1989). In practical applications of this convection phenomenon, the Rayleigh numbers would be highly supercritical. In this paper, we use a numerical procedure to investigate such a convection process up to 20 times the critical value.

Our linear stability analysis of the problem was preceded by a number of similar investigations. Sun (1973) did theoretical and experimental investigations of the problem for small depth ratios. Nield (1977) considered the limiting case of the 
wavenumber approaching zero. Somerton \& Catton (1982) considered the stability of a superposed fluid and porous layer with respect to internal heat generation.

Recently, there have been several nonlinear numerical studies carried out involving combined porous and fluid layers. Poulikakos (1986) treated a problem in which the superposed fluid and porous layers are confined within a box. The ratio of the height of the box to the width was varied between 0.2 and 1.0. Applying a method developed by Beckermann, Ramadhyani \& Viskanta (1986), Poulikakos used the same set of fundamental equations to govern the motion in the fluid and in the porous layer. This is accomplished by using a binary parameter which assumes the value 0 in the fluid region and 1 in the porous region, thus eliminating terms such as the Darcy term in the fluid region. By this formulation, no explicit account needs to be taken of the interface between the fluid and the porous layer. The solutions were obtained by using a finite-volume algorithm. In a number of examples presented with a Darcy number of $10^{-4}$, the streamline patterns show that there is quite a bit of flow penetration from the fluid layer into the porous layer. Nishimura et al. (1986) considered the problem of a superposed layer in a tall, slender box which is heated differentially along the two vertical walls. A finite-element method was used for the solution of the problem. The results compare favourably with experimental results obtained by the same authors. Beckermann et al. (1986) and Beckermann, Viskanta \& Ramadhyani (1988) considered the sideways heating of three variations of combined fluid and porous layers, including a fluid layer on top of a porous layer. Using the one-equation model, a finite-volume method was applied to solve the equations. Results obtained by computation agreed well with those obtained by experiment.

In our investigation, we use the Navier-Stokes equations for the fluid layer and the extended Darcy equation (including Brinkman and Forchheimer terms) for the porous layer. Appropriate boundary conditions are applied at the interface between the fluid and porous layers. The flow is assumed to be two-dimensional and periodic in the horizontal direction. The horizontal extent of the calculation domain is equal to one critical wavelength, as predicted by linear stability theory (Chen \& Chen, 1988). A Galerkin method is applied in the horizontal direction, and a hybrid finitedifference method is used in the vertical direction. The hybrid includes the implicit scheme of Crank-Nicolson for the diffusion terms and the explicit Adam-Bashforth scheme for the nonlinear terms. This numerical algorithm is second-order accurate in both time and space. The computational method is first verified with results obtained by Combarnous \& Bories (1975) and Georgiadis \& Catton (1986) for porous layers, and with the results of the linear stability theory. Then the algorithm was applied to the two-layer system to study the effects of depth ratio, Rayleigh number, and Prandtl number on the characteristics of thermal convection. Results for $\hat{d}=0.1$ and 0.2 are in good agreement with the experimental values found in Chen \& Chen (1989).

\section{Problem formulation}

A porous layer of thickness $d_{\mathrm{m}}$ underlying a fluid layer of thickness $d$ is considered. The top of the fluid layer and the bottom of the porous layer are bounded by rigid walls held at constant temperatures, with the temperature at the bottom boundary higher than that at the top. It is assumed that the flow is laminar, incompressible, and two-dimensional. The physical properties of the fluid are assumed constant, except for the density in the buoyancy term in the momentum equations (Boussinesq approximation). The porous medium is considered homogeneous and isotropic and is 
saturated with a fluid which is in local thermodynamic equilibrium with the solid matrix. A Cartesian coordinate system is chosen with the origin at the interface between the porous and fluid layers, the $z$-axis vertically upwards, and the $y$-axis horizontal. The continuity, momentum, and energy equations for the fluid layer are

$$
\begin{gathered}
\boldsymbol{\nabla} \cdot \boldsymbol{u}=0 \\
\rho_{0}\left(\frac{\partial u}{\partial t}+\boldsymbol{u} \cdot \nabla \boldsymbol{u}\right)=-\nabla P+\mu \nabla^{2} \boldsymbol{u}-\rho_{0} g\left[1-\beta\left(T-T_{0}\right)\right] \\
\left(\rho_{0} C_{p}\right)\left(\frac{\partial T}{\partial t}+u \cdot \nabla T\right)=k \nabla^{2} T .
\end{gathered}
$$

The corresponding equations for the porous layer are (Georgiadis \& Catton 1986)

$$
\begin{gathered}
\boldsymbol{\nabla} \cdot \boldsymbol{u}_{\mathrm{m}}=0 \\
\rho_{0}\left(\frac{1}{\phi} \frac{\partial \boldsymbol{u}_{\mathrm{m}}}{\partial t}+\frac{B}{K}\left|u_{\mathrm{m}}\right| \boldsymbol{u}_{\mathrm{m}}\right)=-\nabla P_{\mathrm{m}}-\frac{\mu}{K} u_{\mathrm{m}}-\rho_{0} g\left[1-\beta\left(T_{\mathrm{m}}-T_{0}\right)\right]+\frac{\mu}{\phi} \nabla^{2} u_{\mathrm{m}} \\
\left(\rho_{0} C_{p}\right)_{\mathrm{m}} \frac{\partial T_{\mathrm{m}}}{\partial t}+\left(\rho_{0} C_{p}\right) u_{\mathrm{m}} \cdot \nabla T_{\mathrm{m}}=k_{\mathrm{m}} \nabla^{2} T_{\mathrm{m}} .
\end{gathered}
$$

In these equations, the subscript $m$ denotes quantities pertaining to the porous medium and $\boldsymbol{u}_{\mathrm{m}}$ denotes the filtration velocity. The reference density of the fluid is denoted by $\rho_{0}$, time by $t$, pressure by $P$, dynamic viscosity by $\mu$, gravitational acceleration by $g$, temperature by $T$, specific heat by $C_{p}$, heat conductivity by $k$, porosity by $\phi$, and the thermal expansion coefficient by $\beta$, which is defined as

$$
\beta=-\frac{1}{\rho_{0}}\left[\frac{\partial \rho}{\partial T}\right]_{p} .
$$

The permeability of the porous medium is denoted by $K$. For a porous medium consisting of glass spheres, $K$ is obtained by the Kozeny-Carmen relation (Combarnous \& Bories 1975),

$$
K=\frac{D^{2}}{172.8} \frac{\phi^{3}}{(1-\phi)^{2}},
$$

in which $D$ is the diameter of the spheres. The heat conductivity of the medium, $k_{\mathrm{m}}$, is calculated by

$$
k_{\mathrm{m}}=\phi k+(1-\phi) k_{\mathrm{g}}
$$

where $k_{\mathrm{g}}$ is the heat conductivity of glass.

In the momentum equation for the porous layer, (5), two additional terms have been included in Darcy's equation. One is the Brinkman term $(\mu / \phi) \nabla^{2} u_{m}$ to account for the viscous effect. This term is only effective in the neighbourhood of solid boundaries and interfaces. The Forchheimer term $(B / K)\left|\boldsymbol{u}_{\mathrm{m}}\right| \boldsymbol{u}_{\mathrm{m}}$ takes into account the inertial effects when the filtration velocity $u_{\mathrm{m}}$ becomes large (see discussion in Nield \& Joseph 1985). The coefficient $B$, which is known as the form drag constant, is independent of the properties of the fluid, but is dependent on the geometry of the medium. For a layer of solid spheres, it can be expressed, according to Ergun (1952), as

$$
B=\frac{1.75 D}{150(1-\phi)}
$$


Generally, the magnitude of the Forchheimer term is relatively small, except when the solid matrix of the porous medium is sparsely distributed. Experimental support for this form of the quadratic drag is described by Ward (1964), while the many experimental results summarized by MacDonald et al. (1979) are consistent with this form. It is noted that the 'effective viscosity' in the porous medium is chosen to be equal to the fluid viscosity (Neale \& Nader, 1974).

The boundary conditions at the top and bottom walls are

$$
\left.\begin{array}{c}
V=W=0, \quad T=T_{\mathrm{u}} \quad \text { at } \quad z=d ; \\
V_{\mathrm{m}}=W_{\mathrm{m}}=0, \quad T_{\mathrm{m}}=T_{\ell} \quad \text { at } \quad z=-d_{\mathrm{m}}
\end{array}\right\}
$$

At the interface, $z=0$, the boundary conditions are the continuities of the velocity, temperature, shear stress, normal stress, and heat flux:

$$
\begin{gathered}
V=V_{\mathrm{m}}, \quad \mu \frac{\partial V}{\partial z}=\mu \frac{\partial V_{\mathrm{m}}}{\partial z} \\
W=W_{\mathrm{m}}, \quad P+2 \mu \frac{\partial W}{\partial z}=P_{\mathrm{m}}+2 \mu \frac{\partial W_{\mathrm{m}}}{\partial z} \\
T=T_{\mathrm{m}}, \quad k \frac{\partial T}{\partial z}=k_{\mathrm{m}} \frac{\partial T_{\mathrm{m}}}{\partial z}
\end{gathered}
$$

To render the equations non-dimensional, the characteristic length is chosen to be the total height, $H=d+d_{\mathrm{m}}$, of the fluid and porous layers, time to be $H^{2} / \lambda$, velocity to be $v / H$, and pressure to be $\rho_{0} \nu^{2} / H^{2}$ for both layers. Different scales for temperatures are used; they are $\left(T_{0}-T_{\mathrm{u}}\right) \nu / \lambda$ and $\left(T_{\ell}-T_{0}\right) \nu / \lambda_{\mathrm{m}}$ for the fluid and porous layers, respectively. After non-dimensionalization, the equations in the fluid layer become

$$
\begin{gathered}
\frac{\partial V}{\partial y}+\frac{\partial W}{\partial z}=0 \\
\frac{1}{\operatorname{Pr}} \frac{\partial V}{\partial t}+V \frac{\partial V}{\partial y}+W \frac{\partial V}{\partial z}=-\frac{\partial P}{\partial y}+\frac{\partial^{2} V}{\partial y^{2}}+\frac{\partial^{2} V}{\partial z^{2}} \\
\frac{1}{\operatorname{Pr}} \frac{\partial W}{\partial t}+V \frac{\partial W}{\partial y}+W \frac{\partial W}{\partial z}=-\frac{\partial P}{\partial z}+\frac{\partial^{2} W}{\partial y^{2}}+\frac{\partial^{2} W}{\partial z^{2}}+\hat{H}^{3} R T \\
\frac{\partial T}{\partial t}+\operatorname{Pr}\left(V \frac{\partial T}{\partial y}+W \frac{\partial T}{\partial z}\right)=\frac{\partial^{2} T}{\partial y^{2}}+\frac{\partial^{2} T}{\partial z^{2}}
\end{gathered}
$$

The equations in the porous layer are

$$
\begin{gathered}
\frac{\partial V_{\mathrm{m}}}{\partial y}+\frac{\partial W_{\mathrm{m}}}{\partial z}=0 \\
\frac{1}{\phi P r} \frac{\partial V_{\mathrm{m}}}{\partial t}+\frac{B H}{K}\left|\boldsymbol{u}_{\mathrm{m}}\right| V_{\mathrm{m}}=-\frac{\partial P_{\mathrm{m}}}{\partial y}-\left(\frac{\hat{H} \hat{d}}{\delta}\right)^{2} V_{\mathrm{m}}+\frac{1}{\phi}\left(\frac{\partial^{2} V_{\mathrm{m}}}{\partial y^{2}}+\frac{\partial^{2} V_{\mathrm{m}}}{\partial z^{2}}\right) \\
\frac{1}{\phi P r} \frac{\partial W_{\mathrm{m}}}{\partial t}+\frac{B H}{K}\left|\boldsymbol{u}_{\mathrm{m}}\right| W_{\mathrm{m}}=-\frac{\partial P_{\mathrm{m}}}{\partial z}-\left(\frac{\hat{H} \hat{d}}{\delta}\right)^{2} W_{\mathrm{m}}+\frac{1}{\phi}\left(\frac{\partial^{2} W_{\mathrm{m}}}{\partial y^{2}}+\frac{\partial^{2} W_{\mathrm{m}}}{\partial z^{2}}\right)+\frac{(\hat{H} \hat{d})^{3}}{\delta^{2}} R_{\mathrm{m}} T_{\mathrm{m}} \\
\epsilon_{T} G_{\mathrm{m}} \frac{\partial T_{\mathrm{m}}}{\partial t}+\operatorname{Pr}_{\mathrm{m}}\left(V_{\mathrm{m}} \frac{\partial T_{\mathrm{m}}}{\partial y}+W_{\mathrm{m}} \frac{\partial T_{\mathrm{m}}}{\partial z}\right)=\frac{\partial^{2} T_{\mathrm{m}}}{\partial y^{2}}+\frac{\partial^{2} T_{\mathrm{m}}}{\partial z^{2}}
\end{gathered}
$$


In these equations, the non-dimensional parameters are defined as follows: $\hat{H}=H / d$; $\hat{d}=d / d_{\mathrm{m}} ; \delta^{2}$ (the Darcy number) $=K / d_{\mathrm{m}}^{2} ; G_{\mathrm{m}}=\left(\rho_{0} C_{p}\right)_{\mathrm{m}} /\left(\rho_{0} C_{p}\right) ;$ and $\epsilon_{T}=\lambda / \lambda_{\mathrm{m}}$, where $\lambda$ is the thermal diffusivity and

$$
\lambda_{\mathrm{m}}=\frac{k_{\mathrm{m}}}{\rho_{0} C_{p}} .
$$

The Prandtl numbers are $\operatorname{Pr}=\nu / \lambda$ and $P r_{\mathrm{m}}=\nu / \lambda_{\mathrm{m}}$, and the Rayleigh numbers defined for the fluid and porous layers are, respectively,

$$
R=\frac{g \beta\left(T_{0}-T_{\mathrm{u}}\right) d^{3}}{\nu \lambda}, \quad R_{\mathrm{m}}=\frac{g \beta\left(T_{\ell}-T_{0}\right) d_{\mathrm{m}} K}{\nu \lambda_{\mathrm{m}}},
$$

in which $T_{0}$ is the temperature at the interface of the heat conduction state. The boundary conditions are

$$
\begin{gathered}
V=W=0, \quad T=\frac{-1}{P r}, \quad \text { at } \quad z=\frac{1}{\hat{H}} ; \\
V_{\mathrm{m}}=W_{\mathrm{m}}=0, \quad T_{\mathrm{m}}=\frac{1}{P r_{\mathrm{m}}}, \quad \text { at } \quad z=-\frac{1}{1+\hat{d}} ; \\
V=V_{\mathrm{m}}, \quad W=W_{\mathrm{m}}, \quad \frac{\partial V}{\partial z}=\frac{\partial V_{\mathrm{m}}}{\partial z}, \quad P=P_{\mathrm{m}} \\
T=T_{\mathrm{m}} \frac{\epsilon_{T}^{2}}{\hat{d}}, \quad \frac{\partial T}{\partial z}=\frac{\partial T_{\mathrm{m}}}{\partial z} \frac{\epsilon_{T}}{\hat{d}}
\end{gathered}
$$

\section{Numerical method of solution}

The two most popular and successful methods for solving systems of partial differential equations (PDEs) arising from convection-diffusion problems are the finite-differencing and the spectral schemes. The spectral methods have always been widely used for natural convection problems. This is because relatively few degrees of freedom are needed to approximate a given function (especially for a smooth function), which reduces both computer storage and execution time. Also, they are relatively easy to implement if the boundary conditions allow the use of trigonometric functions.

In this study, we employ a method which combines the Galerkin and finitedifference methods as developed by Rogers \& Beard (1969) and Meyer-Spache \& Keller (1980) for the Taylor-Couette problem and by McDonough (1980) for the twodimensional Rayleigh-Bénard problem. Georgiadis \& Catton (1986) applied the same scheme as used by McDonough on Bénard convection in a porous medium. Recently, Buell (1988) extended this method to solve the three-dimensional natural convection problem.

The basic idea of these 'mixed' methods is to use the Galerkin method in the direction where it is most efficient or convenient, and to use finite differencing in the other direction. Thus, the dependent variables are expanded in Fourier series in the direction which has a periodic structure, and the Galerkin method is applied. A system of PDEs (differentiated with respect to time and the remaining spatial coordinate) is obtained and approximated by a second-order-accurate centraldifferencing scheme. To deal with systems of nonlinear algebraic equations, MeyerSpache \& Keller (1980) used a full Newton's method, while McDonough (1980) 
performed a modal decoupling. Both methods require tedious iterations and initial guesses, or some ad hoc damping factors, even though the modal decoupling significantly reduces the number of iterations. To avoid the difficulties in initial guesses and enormous numbers of iterations for the initial boundary-value problem in this study, we employ the Adams-Bashforth explicit scheme to deal with the nonlinear terms. This way, the nonlinear terms become forcing terms on the righthand sides of the equations, and the equations for each mode of $W, T$, etc., can be solved separately.

It is assumed that the flow exhibits a horizontal periodicity. Truncated Fourier series which are formed in the functional space spanned by an orthonormal basis are utilized to represent the solution. In the fluid layer

$$
\left[\begin{array}{c}
V(y, z, t) \\
W(y, z, t) \\
T(y, z, t) \\
P(y, z, t)
\end{array}\right]=\left[\begin{array}{c}
0 \\
0 \\
T_{0}(z, t) \\
P_{0}(z, t)
\end{array}\right]+\sum_{K=1}\left[\begin{array}{c}
V_{K}(z, t) \sin \alpha_{K} y \\
W_{K}(z, t) \cos \alpha_{K} y \\
T_{K}(z, t) \cos \alpha_{K} y \\
P_{K}(z, t) \cos \alpha_{K} y
\end{array}\right],
$$

and in the porous layer

$$
\left[\begin{array}{c}
V_{\mathrm{m}}(y, z, t) \\
W_{\mathrm{m}}(y, z, t) \\
T_{\mathrm{m}}(y, z, t) \\
P_{\mathrm{m}}(y, z, t) \\
\left|u_{\mathrm{m}}\right|(y, z, t)
\end{array}\right]=\left[\begin{array}{c}
0 \\
0 \\
\bar{T}_{0}(z, t) \\
\bar{P}_{0}(z, t) \\
\bar{Q}_{0}(z, t)
\end{array}\right]+\sum_{K=1}\left[\begin{array}{c}
\bar{V}_{K}(z, t) \sin \alpha_{K} y \\
\bar{W}_{K}(z, t) \cos \alpha_{K} y \\
\bar{T}_{K}(z, t) \cos \alpha_{K} y \\
\bar{P}_{K}(z, t) \cos \alpha_{K} y \\
\bar{Q}_{K}(z, t) \cos \alpha_{K} y
\end{array}\right],
$$

where $\alpha_{K}=k \alpha$ with $k=1,2,3, \ldots, K$, and $\alpha$ is the non-dimensional critical wavenumber obtained from the linear stability analysis (Chen $\&$ Chen 1988). It is defined in terms of the critical wavelength $l, \alpha=2 \pi H / l$. According to the continuity of the velocities at the interface between the fluid and porous layers, the nondimensional wavenumbers in both layers are equal. To be consistent with our linear analysis, we use the wavenumber $a_{\mathrm{m}}$, which is non-dimensionalized by the thickness of the porous layer $d_{\mathrm{m}}$ in the presentation of results. The relationship between $a_{\mathrm{m}}$ and $\alpha$ is

$$
a_{\mathrm{m}}=2 \pi d_{\mathrm{m}} / l=\alpha /\left(1+d / d_{\mathrm{m}}\right) \text {. }
$$

The existence of periodic solutions to (23) and (24) has been proved by Rabinowitz (1968) for Rayleigh numbers near the onset. McDonough (1980) successfully applied these series for Rayleigh numbers up to highly supercritical ones. By numerous numerical tests, McDonough (1980) and Buell (1988) have studied the convergence rates and the absolute accuracy of the series.

After eliminating the pressures in (12), (13), (16), and (17), we substitute (23) and (24) into the resulting equations, weighted with the appropriate basis, and form inner products (integrate in the $y$-direction over the convection cell), and then obtain a system of Galerkin PDEs :

fluid layer

$$
\begin{gathered}
\frac{1}{\operatorname{Pr}} \frac{\partial}{\partial t}\left(\mathrm{D}^{2}-\alpha_{K}^{2}\right) W_{K}-\left(\mathrm{D}^{2}-\alpha_{K}^{2}\right)^{2} W_{K}=f_{1, K}-f_{2, K} \\
\frac{\partial T_{K}}{\partial t}-\left(\mathrm{D}^{2}-\alpha_{K}^{2}\right) T_{K}=-\operatorname{Pr}_{3, K} \\
-\frac{\partial T_{0}}{\partial t}+\mathrm{D}^{2} T_{0}=\frac{1}{2} \operatorname{Pr} \sum_{i=1} \mathrm{D}\left(W_{i} T_{i}\right)
\end{gathered}
$$


porous layer

$$
\begin{gathered}
\frac{1}{\phi \operatorname{Pr}} \frac{\partial}{\partial t}\left(\mathrm{D}^{2}-\alpha_{K}^{2}\right) \bar{W}_{K}-\frac{1}{\phi}\left(\mathrm{D}^{2}-\alpha_{K}^{2}\right)^{2} \bar{W}_{K}+\left(\frac{\hat{H} \hat{d}}{\delta}\right)^{2}\left(\mathrm{D}^{2}-\alpha_{K}^{2}\right) \bar{W}_{K}=\frac{B H}{K} \bar{f}_{1, K}-\bar{f}_{2, K}, \\
\epsilon_{T} G_{\mathrm{m}} \frac{\partial \bar{T}_{K}}{\partial t}-\left(\mathrm{D}^{2}-\alpha_{K}^{2}\right) \bar{T}_{K}=-\operatorname{Pr}_{\mathrm{m}} \bar{f}_{3, K} \\
-\epsilon_{T} G_{\mathrm{m}} \frac{\partial \bar{T}_{0}}{\partial t}+\mathrm{D}^{2} \bar{T}_{\mathrm{o}}=\frac{1}{2} \operatorname{Pr}_{\mathrm{m}} \sum_{i=1} \mathrm{D}\left(\bar{W}_{i} \bar{T}_{i}\right)
\end{gathered}
$$

The boundary conditions become:

$$
\begin{gathered}
T_{0}=\frac{-1}{P r}, \quad T_{K}=W_{K}=W_{K}^{\prime}=0 \quad \text { at } z=1 / \hat{H} \\
\bar{T}_{0}=\frac{1}{P r_{\mathrm{m}}}, \quad \bar{T}_{K}=\bar{W}_{K}=\bar{W}_{K}^{\prime}=0 \quad \text { at } z=-1 /(1+d) ; \\
W_{K}=\bar{W}_{K}, \quad W_{K}^{\prime}=\bar{W}_{K}^{\prime}, \quad W_{K}^{\prime \prime}=\bar{W}_{K}^{\prime \prime}, \\
T_{0}=\frac{\epsilon_{T}^{2}}{\hat{d}} \bar{T}_{0}, \quad \bar{T}_{K}=\frac{\epsilon_{T}^{2}}{\hat{d}} \bar{T}_{K}, \quad T_{0}^{\prime}=\frac{\epsilon_{T}}{\hat{d}} \bar{T}_{0}^{\prime}, \quad T_{K}^{\prime}=\frac{\epsilon_{T}}{\hat{d}} \bar{T}_{K}^{\prime}, \quad \text { at } \quad z=0 ; \\
-\frac{1}{P r} \frac{\partial W_{K}^{\prime}}{\partial t}+\alpha_{K} f_{4, K}+\left(W_{K}^{\prime \prime \prime}-\alpha_{K}^{2} W_{K}^{\prime}\right)=\frac{-1}{\phi P r} \frac{\partial \bar{W}_{K}^{\prime}}{\partial t}+\frac{B H}{K} \alpha_{K} \overline{f_{4, K}} \\
+\frac{1}{\phi}\left(\bar{W}_{K}^{\prime \prime \prime}-\alpha_{K}^{2} \bar{W}_{K}^{\prime}\right)-\left(\frac{\hat{H} \hat{d}}{\delta}\right)^{2} \bar{W}_{K}^{\prime},
\end{gathered}
$$

in which $f_{i, K}, i=1, \ldots, 4$ are forcing terms in the fluid layer,

$$
\begin{array}{r}
f_{1, K}=\sum_{i=1} \sum_{j=1}\left\{\frac{\alpha_{K}}{\alpha_{i}} I_{1}(k, i, j)\left(W_{i}^{\prime \prime} W_{j}^{\prime}+W_{i}^{\prime} W_{j}^{\prime \prime}\right)-\frac{\alpha_{K}}{\alpha_{j}} I_{1}(k, j, i)\left(W_{i}^{\prime} W_{j}^{\prime \prime}+W_{i} W_{j}^{\prime \prime \prime}\right)\right. \\
\left.+\alpha_{K}^{2}\left[\frac{\alpha_{j}}{\alpha_{i}} I_{1}(i, j, k) W_{i}^{\prime} W_{j}+I_{2}(k, i, j) W_{i} W_{j}^{\prime}\right]\right\}, \\
f_{2, K}=\alpha_{K}^{2} \hat{H}^{3} R T_{K}, \\
f_{3, K}=T_{0}^{\prime} W_{K}+\sum_{i=1} \sum_{j=1}\left[\frac{\alpha_{j}}{\alpha_{i}} I_{1}(i, j, k) W_{i}^{\prime} T_{j}+I_{2}(k, i, j) W_{i} T_{j}^{\prime}\right], \\
f_{4, K}=\sum_{i=1} \sum_{j=1}\left[\frac{I_{1}(k, i, j)}{\alpha_{i}} W_{i}^{\prime} W_{j}^{\prime}-\frac{I_{1}(k, j, i)}{\alpha_{j}} W_{i} W_{j}^{\prime \prime}\right]
\end{array}
$$

and $\bar{f}_{i, K}, i=1, \ldots, 4$, are the counterparts in the porous layer,

$$
\begin{gathered}
\bar{f}_{1, K}=-\bar{Q}_{0}\left(\bar{W}_{K}^{\prime \prime}-\alpha_{K}^{2} \bar{W}_{K}\right)-\bar{Q}_{0}^{\prime} \bar{W}_{K}^{\prime}+\sum_{i=1} \sum_{j=1}\left[\alpha_{K}^{2} I_{2}(k, i, j) \bar{W}_{i} \bar{Q}_{j}\right. \\
\left.-\frac{\alpha_{K}}{\alpha_{i}} I_{1}(k, i, j)\left(\bar{W}_{i}^{\prime \prime} \bar{Q}_{j}+\bar{W}_{i}^{\prime} \bar{Q}_{j}^{\prime}\right)\right] \\
\bar{f}_{2, K}=\alpha_{K}^{2} \hat{H}^{3} R_{\mathrm{m}} \bar{T}_{K} \\
\bar{f}_{3, K}=\bar{T}_{0}^{\prime} \bar{W}_{K}+\sum \sum_{i=1}\left[\frac{\alpha_{j}}{\alpha_{i}} I_{1}(i, j, k) \bar{W}_{i}^{\prime} \bar{T}_{j}+I_{2}(k, i, j) \bar{W}_{i} \bar{T}_{j}^{\prime}\right] \\
\bar{f}_{4, K}=-\bar{Q}_{0} \frac{\bar{W}_{K}^{\prime}}{\alpha_{K}}-\sum_{i=1} \sum_{j=1} \bar{Q}_{i} \frac{W_{j}^{\prime}}{\alpha_{j}} I_{1}(k, j, i) .
\end{gathered}
$$


The $\mathrm{D}$ in (25) to (30) and the prime in subsequent equations denote the spatial derivative in the $z$-direction. The convolution products $I_{1}$ and $I_{2}$ are

$$
\begin{gathered}
I_{1}(k, i, j)=\frac{2 \alpha}{\pi} \int_{0}^{\pi / \alpha} \sin \alpha_{K} y \sin \alpha_{i} y \cos \alpha_{j} y \mathrm{~d} y=\left\{\begin{array}{cc}
0.5 & j=|k+i|, \\
-0.5 & j=k+i, \\
0 & \text { otherwise; }
\end{array}\right. \\
I_{2}(k, i, j)=\frac{2 \alpha}{\pi} \int_{0}^{\pi / \alpha} \cos \alpha_{K} y \cos \alpha_{i} y \cos \alpha_{j} y \mathrm{~d} y=\left\{\begin{array}{cc}
0.5, & j=|k-i| \\
& j=k+i, \\
0 & \text { otherwise. }
\end{array}\right.
\end{gathered}
$$

The quadratic Forchheimer terms in (38) and (40), i.e. $\left|\boldsymbol{u}_{\mathrm{m}}\right|$, are evaluated first in the physical plane using the relation $\left|\boldsymbol{u}_{\mathrm{m}}\right|=\left(v_{\mathrm{m}}^{2}+w_{\mathrm{m}}^{2}\right)^{\frac{1}{2}}$ and then transformed to the spectral plane to evaluate $Q_{K}$ by using a discretized fast Fourier transform (FFT) for each time step.

For solving nonlinear convection-diffusion equations in one spatial dimension, Peyret \& Taylor (1982) suggested the Crank-Nicolson, Adams-Bashforth hybrid scheme when diffusion (viscosity or thermal diffusivity) is not too small. This hybrid implicit/explicit method is quite efficient computationally because the nonlinear terms are handled explicitly and, thus, iteration is avoided. In addition, the severe time-step restriction characteristic of the explicit method is relaxed by advancing the diffusion terms implicitly (Crank-Nicolson). To illustrate the computation procedures more clearly, we choose (25) as a typical example to discretize as follows:

$$
\underbrace{\frac{1}{P r} \frac{\mathrm{L}_{K}^{n+1} W_{K}-\mathrm{L}_{K}^{n} W_{K}}{\Delta t}}_{\text {Forward time step }}-\underbrace{\frac{1}{2}\left[\mathrm{~L}_{K}^{n+1} \mathrm{~L}_{K}^{n+1} W_{K}+\mathrm{L}_{K}^{n} \mathrm{~L}_{K}^{n} W_{K}\right.}_{\text {Crank-Nicolson }}]=[\underbrace{\left.C_{1} f_{1, K}^{n}-C_{2} f_{1, K}^{n-1}\right]}_{\text {Adams-Bashforth }}-f_{2, K}^{n},
$$

where $\mathrm{L}_{K}$ is the differential operator $\mathrm{D}^{2}-\alpha_{K}^{2}$. At $t=0$, let $C_{1}=1$ and $C_{2}=0$ so that the nonlinear terms are advanced initially by the forward Euler method. Thereafter, $C_{1}=1.5$ and $C_{2}=0.5$ are used on the right-hand side of (43) to the end of the computation (usually the steady state). The spatial derivatives in the $z$-direction are approximated by standard central finite differencing. This scheme is second-order accurate in space and time.

Calculations are initiated by a sinusoidal temperature distribution with maximum magnitude $10^{-4}$. Three other types of perturbations have been tried. These are sinusoidal perturbations 2 and 10 times the wavenumber, as initially assumed, and a random perturbation. The final steady states obtained by all four initial perturbations are exactly the same as measured by the Nusselt number and the kinetic energy of the fluid. Only the time to reach steady state corresponding to each perturbation is different. For all subsequent calculations, the sinusoidal perturbation with one complete wave in each layer is used. The number of terms used in the Fourier expansion depends on the depth ratio and Rayleigh number. It ranges from 5 to 15 terms. The computation was carried out on a SCS 40 mini-supercomputer at the University of Arizona and on a CRAY-XMP at the San Diego Supercomputer Center.

\section{Results and discussion}

For the results reported in this section, calculations are made for the following set of parameters: $\phi=$ porosity $=0.389 ; \delta^{2}=$ Darcy number $=0.889 \times 10^{-5} ; \epsilon_{T}=$ 
diffusivity ratio $=0.725 ; G_{\mathrm{m}}=$ specific heat ratio $=0.69$. These values are suitable for a water-saturated porous medium consisting of $3 \mathrm{~mm}$ diameter glass beads.

Before we used the computer code to calculate the convection characteristics of the two-layer system, we applied it to two known cases for verification. One case is for $\hat{d}=0$, the porous layer alone, and the other is for the determination of the critical condition for the onset of convection in a two-layer system.

\subsection{Verification of the method of calculation}

\subsubsection{Convergence and stability of the computation scheme}

The convergence of the hybrid scheme was tested in the following way. For $\hat{d}=1.0$, $\operatorname{Pr}=6.26$, and $R_{\mathrm{m}}=3 R_{\mathrm{m}_{\mathrm{c}}}$, the Nusselt number is evaluated with varying numbers of terms and grid points. First, we fixed the number of grid points at 50 in each of the layers. The calculation yields a Nusselt number of 1.329 for three terms and 1.335 for five or seven terms. It is seen that five terms of the Fourier series are sufficient to represent the solution correctly. We then used the five-term representation and systematically varied the number of grid points in both layers from 30-30 to 100-100 points. The results are shown in table 1 .

It is found that, for the case $\hat{d}=1, \operatorname{Pr}=6.26$, and $R_{\mathrm{m}}=3 R_{\mathrm{m}_{\mathrm{c}}}$, the solution converges with an approximate second-order rate. The same results were observed by McDonough (1980) when this hybrid scheme was applied to a single fluid layer. The number of grid points used for each depth ratio is shown in table 2. Further information about the computational parameters is presented in $\$ 4.2 .1$.

The usual von Neumann stability analysis is not applicable because of the inherent nonlinearity in the governing equations. A complete analysis of the stability of the system represented by (1)-(8) and their respective boundary conditions, (10)-(12), would be an enormous task. However, from a numerical determination of the stable region in the parameter space, Pruett (1986) found, for this hybrid scheme, that as the finite-difference grid is refined (corresponding to a higher-Prandtl-number fluid in our system), smaller time steps are required.

In this study, the number of grid points and the corresponding time steps used were determined by trial and error. In general, a finer grid and smaller time steps are needed and convergence of the Fourier series deteriorates for cases with smaller $\hat{d}$ or higher $R_{\mathrm{m}}$. A detailed discussion of the convergence of the hybrid scheme can be found in McDonough (1980). Truncation of the series at the values of $N$ shown in table 2 leads to errors of about $2 \%$ in the Nusselt number, as determined by comparison with results for larger $N$. Table 2 summarizes the computational parameters used in the current study.

\subsubsection{Convection in a porous layer}

The case of $\hat{d}=0$ with $\operatorname{Pr}_{\mathrm{m}}=4.5$ serves as the first test of the accuracy of the numerical algorithm. It is known that the fluid remains stationary below the first critical Rayleigh number $R_{\mathrm{m}}=4 \pi^{2}$ (Lapwood 1948), with heat transferred by conduction alone. Above this value of $R_{\mathrm{m}}$, steady convection prevails until the second critical value is reached when oscillatory motion first appears. Our computation yields a value for the second critical Rayleigh number between of 390 and 400. Caltagirone (1975) used a finite-difference scheme for flow in a square cell and found that the value of the second critical $R_{\mathrm{m}}$ is $384 \pm 5$, whereas the value reported by Schubert \& Straus (1982) is between 380 and 400 . Steen \& Aidun (1988) obtained a value of 390.7 using an eigenfunction expansion method. Our computations for $R_{\mathrm{m}}=480$ (see figure 1) show that the Nusselt number oscillates 


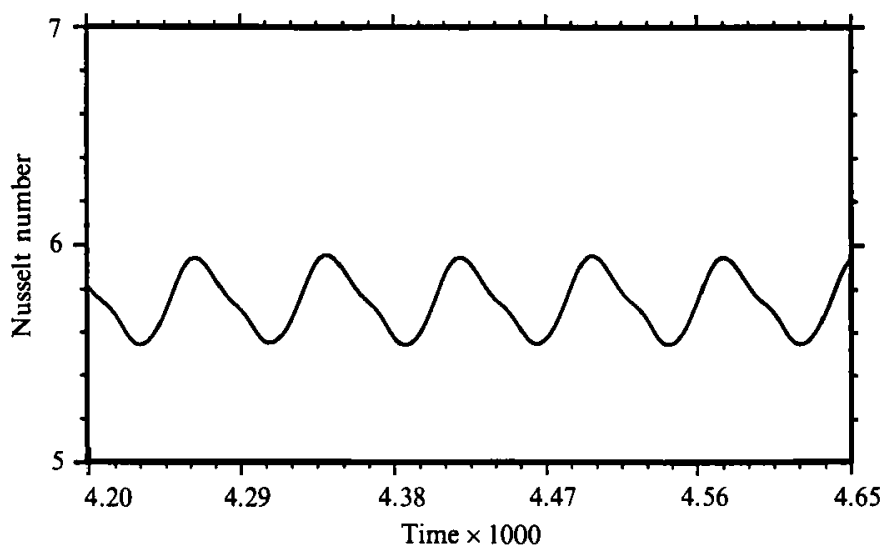

Fraure 1. Oscillation in the Nusselt number in a porous layer $(\hat{d}=0)$ at $R_{\mathrm{m}}=480$ and $P r_{\mathrm{m}}=4.5$.

\begin{tabular}{llllllll}
\hline Points & $30-30$ & $40-40$ & $50-50$ & $60-60$ & $70-70$ & $80-80$ & $100-100$ \\
$N u$ & 1.310 & 1.324 & 1.335 & 1.343 & 1.349 & 1.353 & 1.355 \\
Error (\%) & 3.3 & 2.3 & 1.5 & 0.88 & 0.44 & 0.15 & 0
\end{tabular}

TABLE 1. Effect of varying the number of grid points

\begin{tabular}{|c|c|c|c|c|c|}
\hline$R_{\mathrm{m}} / R_{\mathrm{m}_{\mathrm{c}}}$ & $\hat{d}$ & $\begin{array}{c}\text { Grid-points } \\
\text { (fluid-porous) }\end{array}$ & $\begin{array}{l}\text { No. of } \\
\text { terms }\end{array}$ & $\Delta t$ & $\begin{array}{l}\text { No. of } \\
\text { time steps }\end{array}$ \\
\hline 1.5 & $\left\{\begin{array}{l}0.1 \\
0.2 \\
0.5 \\
1.0\end{array}\right.$ & $\begin{array}{l}50-500 \\
60-120 \\
40-80 \\
50-50\end{array}$ & $\begin{array}{l}5 \\
5 \\
9 \\
6\end{array}$ & $\begin{array}{l}1 \times 10^{-3} \\
5 \times 10^{-4} \\
3 \times 10^{-4} \\
1 \times 10^{-3}\end{array}$ & $\begin{array}{c}1800 \\
300 \\
650 \\
300\end{array}$ \\
\hline 3.0 & $\left\{\begin{array}{l}0.1 \\
0.2 \\
0.5 \\
1.0\end{array}\right.$ & $\begin{array}{l}50-500 \\
60-120 \\
40-80 \\
50-50\end{array}$ & $\begin{array}{l}9 \\
5 \\
5 \\
5\end{array}$ & $\begin{array}{l}1 \times 10^{-4} \\
3 \times 10^{-4} \\
1 \times 10^{-3} \\
1 \times 10^{-3}\end{array}$ & $\begin{array}{c}1600 \\
50 \\
120 \\
120\end{array}$ \\
\hline 5.0 & $\left\{\begin{array}{l}0.1 \\
0.2 \\
0.5 \\
1.0\end{array}\right.$ & $\begin{array}{l}50-500 \\
60-120 \\
40-80 \\
50-50\end{array}$ & $\begin{array}{l}9 \\
7 \\
5 \\
5\end{array}$ & $\begin{array}{l}1 \times 10^{-4} \\
2 \times 10^{-4} \\
3 \times 10^{-4} \\
1 \times 10^{-3}\end{array}$ & $\begin{array}{r}1100 \\
70 \\
120 \\
80\end{array}$ \\
\hline 10.0 & $\left\{\begin{array}{l}0.1 \\
0.2 \\
0.5 \\
1.0\end{array}\right.$ & $\begin{array}{l}50-500 \\
60-120 \\
40-80 \\
50-50\end{array}$ & $\begin{array}{r}12 \\
7 \\
5 \\
5\end{array}$ & $\begin{array}{l}3 \times 10^{-5} \\
5 \times 10^{-5} \\
2 \times 10^{-4} \\
3 \times 10^{-4}\end{array}$ & $\begin{array}{r}1200 \\
70 \\
90 \\
90\end{array}$ \\
\hline 20.0 & $\left\{\begin{array}{l}0.1 \\
0.2 \\
0.5 \\
1.0\end{array}\right.$ & $\begin{array}{l}50-500 \\
60-120 \\
40-80 \\
50-50\end{array}$ & $\begin{array}{r}12 \\
7 \\
5 \\
5\end{array}$ & $\begin{array}{l}2 \times 10^{-5} \\
3 \times 10^{-5} \\
1 \times 10^{-4} \\
2 \times 10^{-4}\end{array}$ & $\begin{array}{r}1200 \\
60 \\
80 \\
50\end{array}$ \\
\hline
\end{tabular}

TABLE 2. Computational parameters used in the current study

with a non-dimensional period of 0.0069 , which compares well with the value of 0.0073 (using our timescale) obtained by Schubert \& Straus. The Nusselt number fluctuates between 5.54 and 5.92 in the current study and between 5.61 and 5.95 in the work of Schubert \& Straus. Further checks of the computer code were made for steady-state cases, i.e. $R_{\mathrm{m}}$ less than 380 . 


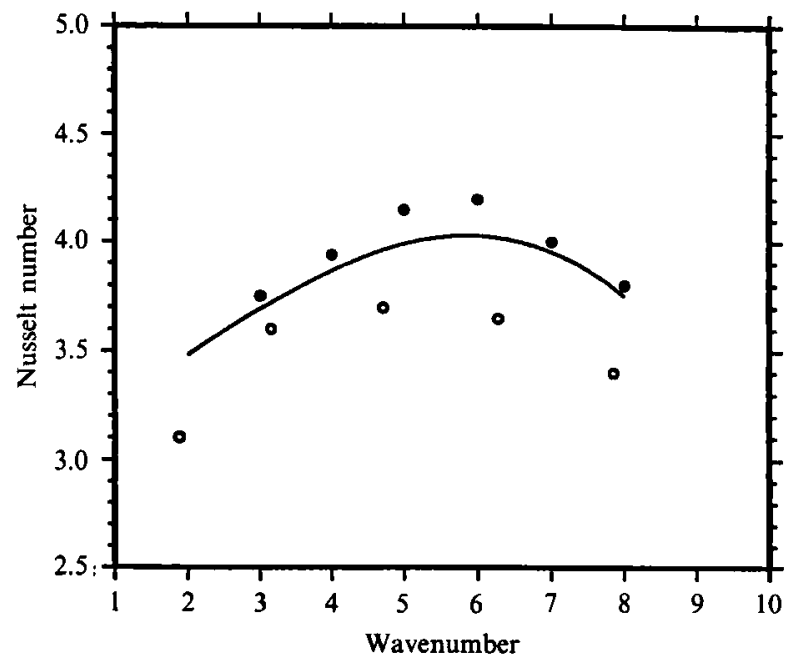

FIGURe 2. Variation of the Nusselt number with wavenumber $a_{\mathrm{m}}$ for a porous layer $(\hat{d}=0)$ at $R_{\mathrm{m}}=200:-$, present results;, Georgiadis \& Catton (1986); $\bigcirc$, Combarnous \& Bories (1975).

Caltagirone (1975)

Schubert \& Straus (1982)

Combarnous \& Bories (1975), fig. 43

Present study

(No. of terms used)

\begin{tabular}{ccccc}
\multicolumn{5}{c}{$R_{\mathrm{m}}$} \\
\hline 100 & 200 & 300 & 350 & 380 \\
2.651 & 3.813 & 4.523 & - & - \\
- & - & - & 4.79 & 4.94 \\
2.6 & 3.8 & 4.5 & - & - \\
2.62 & 3.84 & 4.59 & 4.89 & 5.03 \\
$(5)$ & $(5)$ & $(10)$ & $(15)$ & $(15)$
\end{tabular}

TABLE 3. Comparison of steady-state Nusselt numbers calculated by the present method with those obtained by previous investigators

We compare our results at $R_{\mathrm{m}}=100,200$, and 300 with the computed results of Combarnous \& Bories (1975) and Caltagirone (1975), and at $R=350$ and 380 with those obtained by Schubert \& Straus (1982). The agreement is within $2 \%$, as shown in table 3. It is noted that the number of terms necessary for the Galerkin series to yield correct results increases with the Rayleigh number. A detailed discussion of the influence of the number of terms on the results can be found in Schubert \& Straus.

It is known that as $R_{\mathrm{m}}$ is increased into the supercritical range, the maximum heat transfer is accomplished with cells of smaller wavelengths. We have computed the Nusselt number over a range of wavenumbers from 2 to 8 at $R_{\mathrm{m}}=200$. These values are compared with those obtained by Combarnous \& Bories (1975) and by Georgiadis \& Catton (1986) in figure 2. It is seen that agreement between our results and those of Georgiadis \& Catton are quite good, and that our results are generally higher than those of Combarous \& Bories, but with similar trends.

\subsubsection{Onset of convection in a two-layer system}

We determine the onset of convection using the nonlinear program by monitoring the time evolution of the kinetic energy of the system due to an initial perturbation of infinitesimal magnitude. At subcritical Rayleigh numbers, after the initial disturbance, the kinetic energy decays indefinitely, whereas at supercritical Rayleigh numbers, the kinetic energy grows to large magnitudes. At the critical Rayleigh 


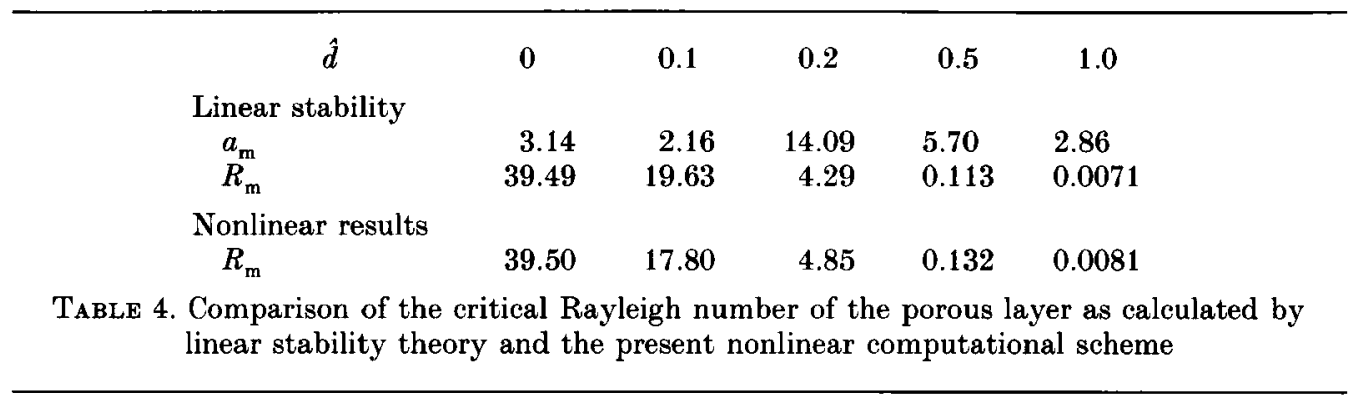

number, the kinetic energy remains constant at a rather low level. The critical Rayleigh numbers thus determined for $\hat{d}=0,0.1,0.2,0.5$, and 1.0 are compared with the corresponding values obtained by Chen \& Chen (1988) using a linear stability analysis in table 4 . The agreement is generally good, with a discrepancy of less than $15 \%$. It is noted, however, that the boundary conditions at the interface used in the nonlinear calculation are different from those used in the linear stability analysis. In the former, regular boundary conditions are invoked at the interface because Darcy's equation is augmented by the Brinkman term. In the latter, a velocity slip is applied at the interface according to the Beavers \& Joseph (1967) condition because only Darcy's equation is used in the porous layer. The discrepancy in the predictions may be attributed to the difference in the boundary conditions. The critical wavenumber as predicted by the linear stability theory for each depth ratio is also listed in table 4 for later reference. It is seen that the wavenumber increased sevenfold between $\hat{d}=0.1$ and 0.2 .

\subsection{Two-layer system with $P r=6.26$}

\subsubsection{Flow patterns and isotherms}

The first set of computations, in which we justify the use of the critical wavelength at onset of convection in all supercritical states, was made for $\hat{d}=1.0$. The steadystate Nusselt number was calculated for cells of decreasing size corresponding to a range of wavenumbers $a_{\mathrm{m}}$ from 2 to 6 at 1.5 to 20 times the critical Rayleigh number. The results are shown in figure 3 . The critical wavenumber according to the linear theory for this case is 2.86 . It is seen from figure 3 that the maximum of each curve is quite shallow; at $a_{\mathrm{m}}=2.86$, the Nusselt number is either at or very close to the maximum value. In view of these results, it is reasonable to assume that the characteristics of convection at the critical wavenumber are representative of the flow in the two-layer system. Therefore, in all subsequent calculations, we fix the size of the convection cell to that at onset for all supereritical Rayleigh numbers.

The streamline patterns and isotherms for $\hat{d}=1.0$ at $R_{\mathrm{m}}=1.5 R_{\mathrm{m}_{\mathrm{c}}}$ up to $20 R_{\mathrm{m}_{\mathrm{c}}}$ are presented in figure 4 . The convection cell is presented at the correct aspect ratio. It is seen that convection is mainly confined within the fluid layer and intensifies as $R_{\mathrm{m}}$ is increased. Furthermore, the convection cell in the fluid layer is located slightly above the porous-fluid interface indicated by the dotted line. The flow in the porous layer is very weak; the streamline patterns are not discernible with $\Delta \psi=0.4$. As $R_{\mathrm{m}}$ is increased, the hot plume rising in the fluid layer becomes stronger. Because of the tangential flow boundary condition at the interface, a weak descending plume is induced in the porous layer.

The results for $\hat{d}=0.5$ are shown in figure 5 . The general trend is similar to the case where $\hat{d}=1.0$. As the convective motion becomes more intense, the temperature difference between the top wall and interface becomes less. At the same time, the 


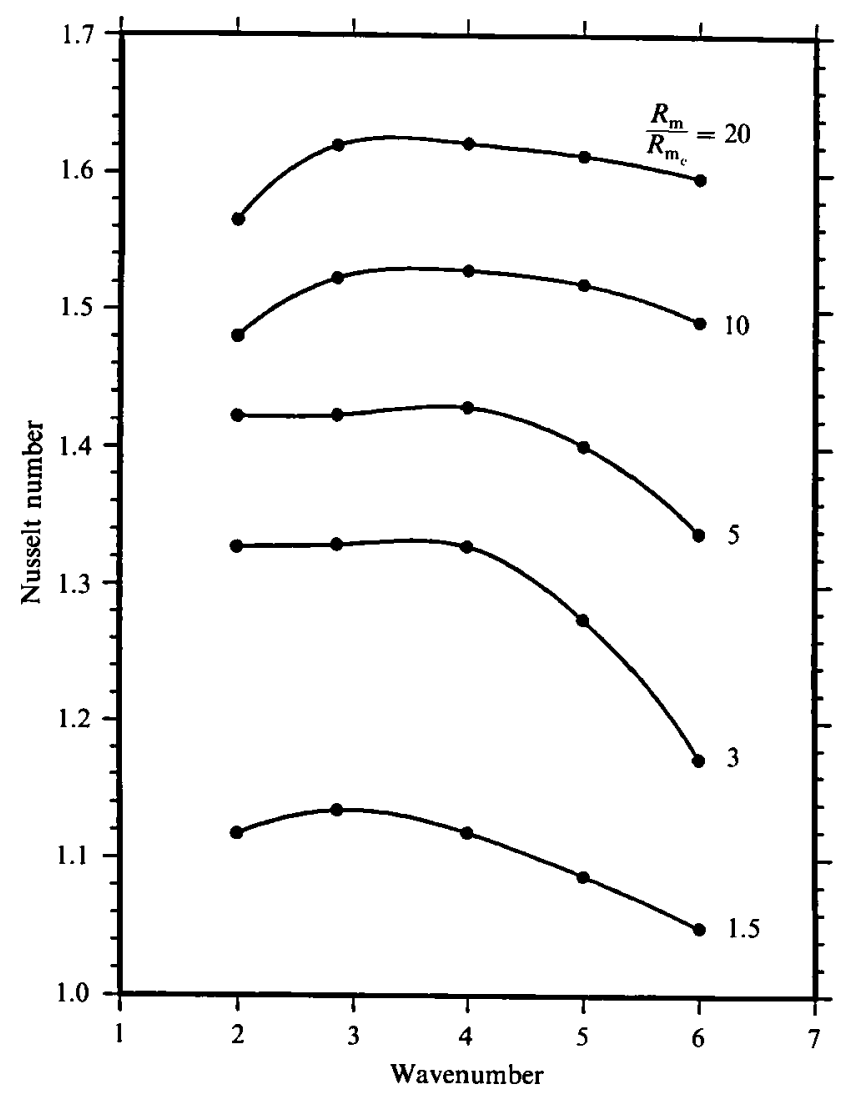

Figure 3. Variation of Nusselt number with wavenumber $a_{\mathrm{m}}$ for $\hat{d}=1.0, \operatorname{Pr}=6.26$ at $R_{\mathrm{m}}=1.5 R_{\mathrm{m}_{\mathrm{c}}}$ to $20 R_{\mathrm{m}_{\mathrm{c}}}$.

temperature difference across the porous layer becomes larger. The upward displacement of the convection cell in the fluid layer becomes more prominent. This is reminiscent of the convection pattern at onset (Chen \& Chen 1988), as shown in figure 6 for $\hat{d}=0.5$ and 1.0 .

As $\hat{d}$ is reduced to 0.2 , the aspect ratio of the unit convection cell becomes quite small, as shown in figure 7. A more intense pair of convection cells is induced in the porous layer at the interface at high $R_{\mathrm{m}}$. At $\hat{d}=0.1$, which is below the critical value of 0.13 , the convection becomes dominated by the porous layer, as shown in figure 8 , consistent with our linear results. The isotherms indicate a downward plume in the middle of the cell, rather than an upward plume as indicated in the previous cases. This is a random occurrence involving a lateral shift of half a wavelength., As $R_{\mathrm{m}}$ is increased, the strength of the plume becomes larger such that it has considerable lateral spread when it reaches the bottom.

For all cases considered, no oscillation was observed up to $20 R_{\mathrm{m}_{\mathrm{c}}}$. It is known that for a porous layer, oscillation starts at $10 R_{\mathrm{m}_{\mathrm{c}}} \approx 380-390$. For a fluid layer, Krishnamurti (1970) has determined experimentally that oscillatory convection starts at $18 R_{\mathrm{c}}$ for fixed-fixed boundary conditions. Recent numerical results of Goldhirsch, Pelz \& Orszag (1989) for convection in a box show that oscillation starts at approximately $20 R_{\mathrm{c}}$. In an infinite horizontal fluid layer with free-free conditions, Curry et al. (1984) showed by calculation that oscillation starts at $50 R_{\mathrm{c}}$ when 

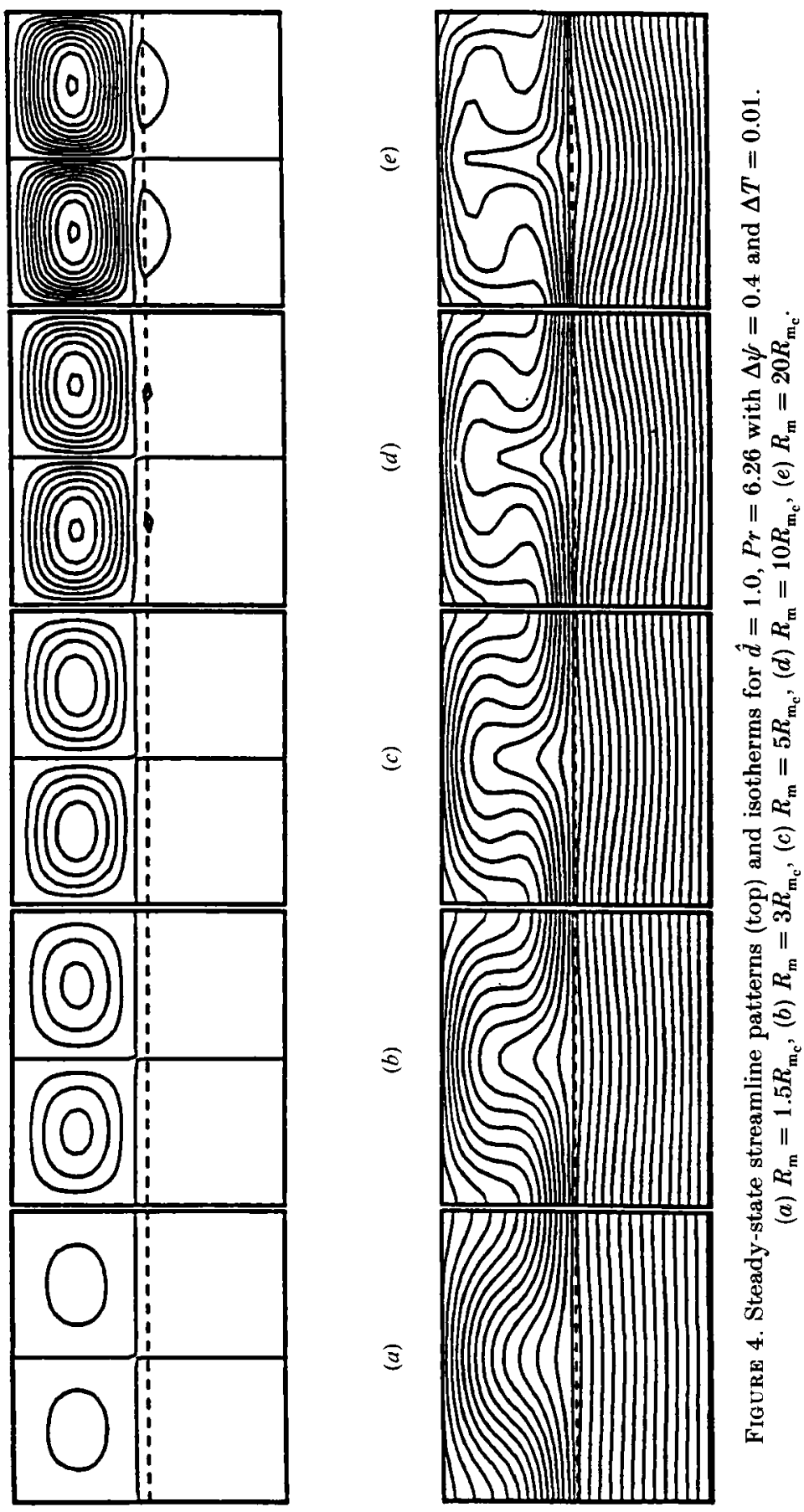

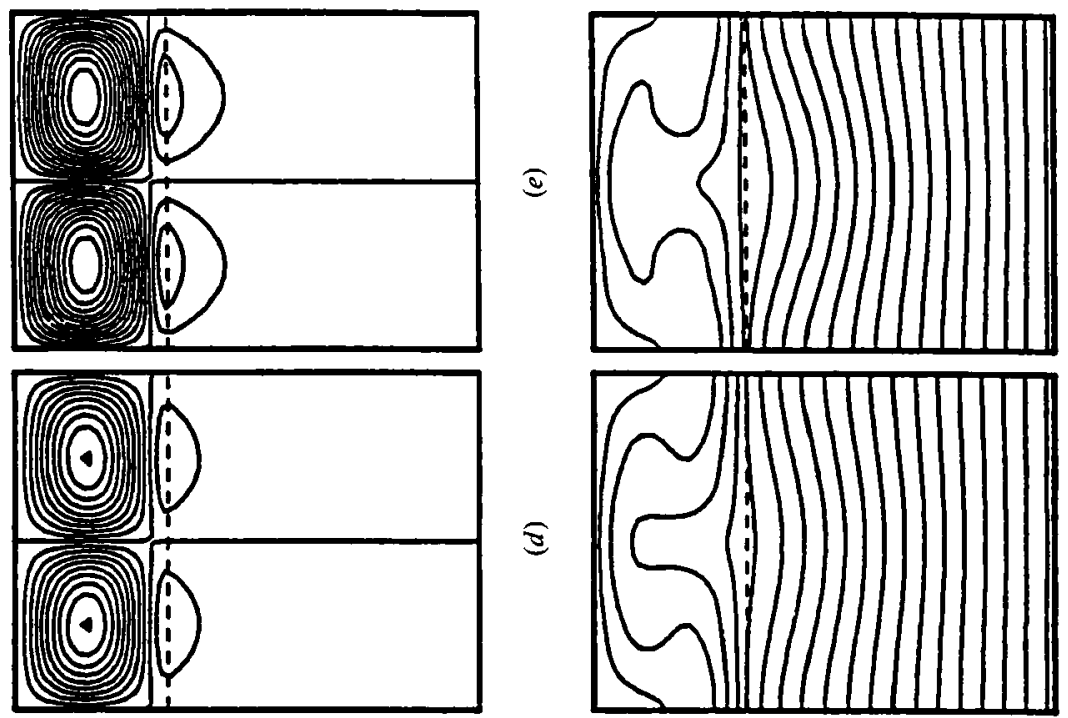

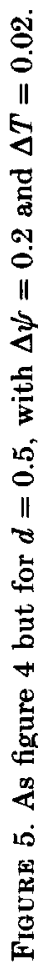
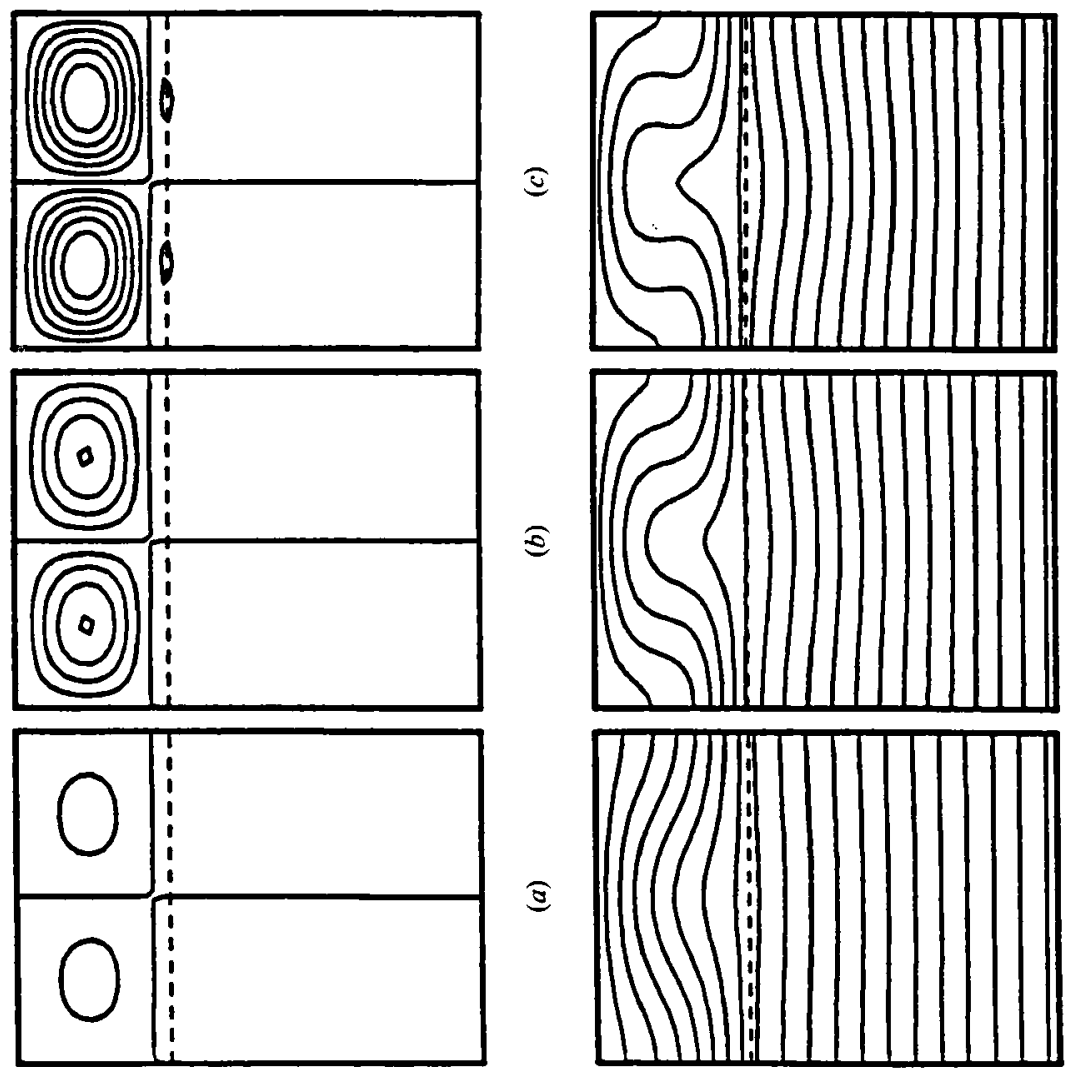
(a)

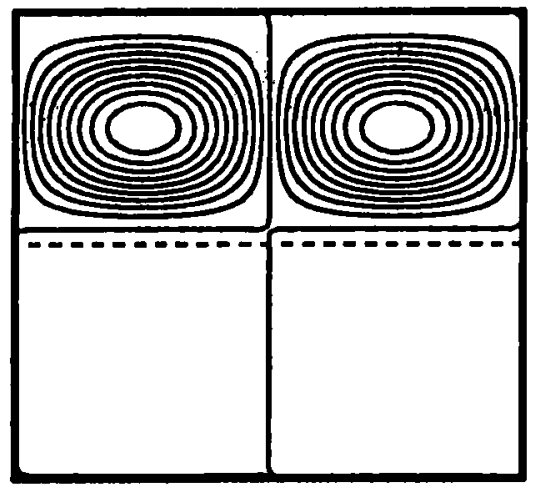

(b)

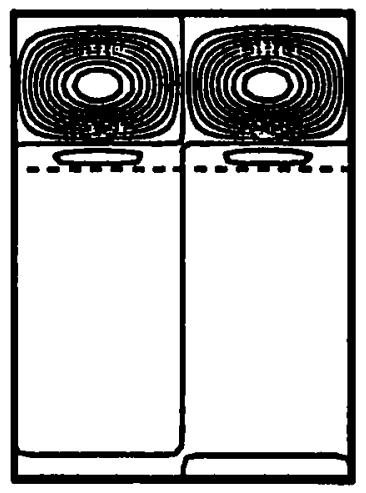

FiquRe 6. Streamline patterns for $(a) \hat{d}=1.0$ and $(b) \hat{d}=0.5$ at onset according to the linear stability theory (Chen \& Chen 1988).

symmetry breaking is permitted. But when symmetry is enforced, transition to oscillatory motion is delayed until $90 R_{\mathrm{c}}$.

The critical Rayleigh numbers in the porous layer, $R_{\mathrm{m}_{\mathrm{c}}}$, and in the fluid layer, $R_{\mathrm{c}}$, are listed in table 5 for four depth ratios of $\hat{d}=0.1$ to 1.0 . For $\hat{d}=0.1$, the stability of the system is dominated by the porous layer. The corresponding $R_{\mathrm{c}}$ is within the subcritical range for a fluid layer. For $\hat{d} \geqslant 0.2$, the stability is controlled by the fluid layer and the values of $R_{\mathrm{c}}$ are comparable to those of a single fluid layer. For all depth ratios considered, $20 R_{\mathrm{m}_{\mathrm{c}}}$ is always less than 390 , the second critical Rayleigh number for transition to oscillatory convection for a porous layer. At larger depth ratios, 0.2 to 1.0 , even though the values of $20 R_{\mathrm{c}}$ are comparable to the second critical Rayleigh number for a fluid layer, we observed no oscillation because of the strong damping effect of the porous layer underneath the fluid layer.

Our procedure of computation is, first to fix the grid points to 50 in each layer, then increase the number of Galerkin terms until convergence is achieved. Then, the grid points in each layer are varied systematically to effect convergence. If no satisfactory convergence is found, the number of terms is increased and the procedure is repeated until satisfactory convergence is obtained. Should the final convection state lie below the second transition point, a steady result emerges. The steady solution is generally followed for approximately $10 \%$ of the time needed to reach the steady solution. Should the final state lie above the second transition point, an unsteady state results. For all cases encountered at $R_{\mathrm{m}}<20 R_{\mathrm{m}_{\mathrm{c}}}$, no oscillatory states were found. We are confident that these results are correct, and they are supported by our physical reasoning presented above. Once oscillatory convection sets in, the number of Galerkin terms increases dramatically because both lower and high modes play a crucial role in the solution, with concomitant increases in CPU time. We did not pursue the determination of the onset of oscillations in the two-layer problem because of constraints on the CPU time available to us.

\subsubsection{Heat transfer across the two-layer system}

The heat transfer across the combined system as represented by the Nusselt number for the cases considered are shown as a function of the Rayleigh number in figure 9 . The values for $\hat{d}=0$ were calculated up to $R_{\mathrm{m}}=390$, beyond which the Nusselt number becomes oscillatory. The Nusselt number for $\hat{d}=0.1$ follows the $\hat{d}=0$ curve 

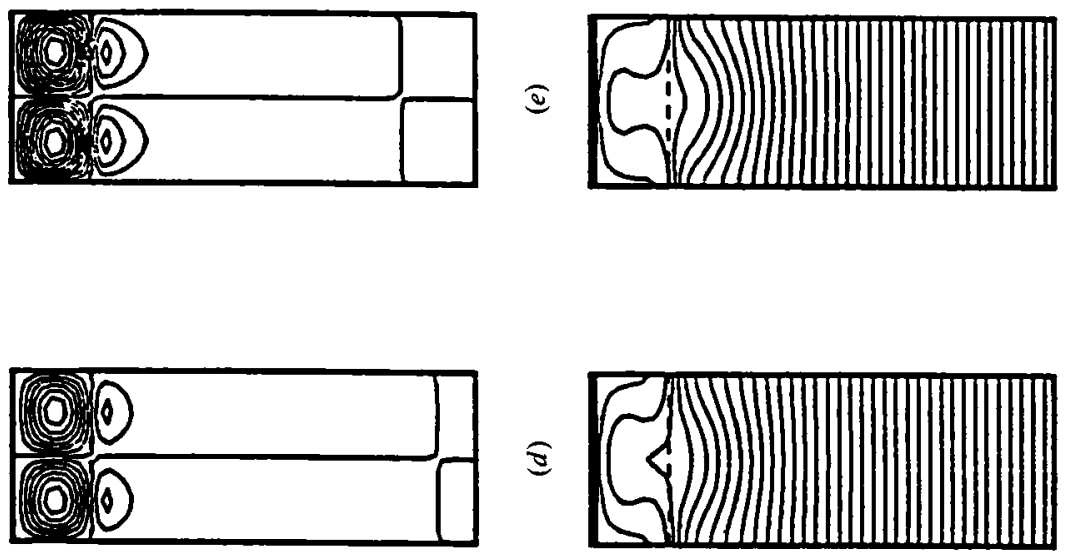

ป⿱
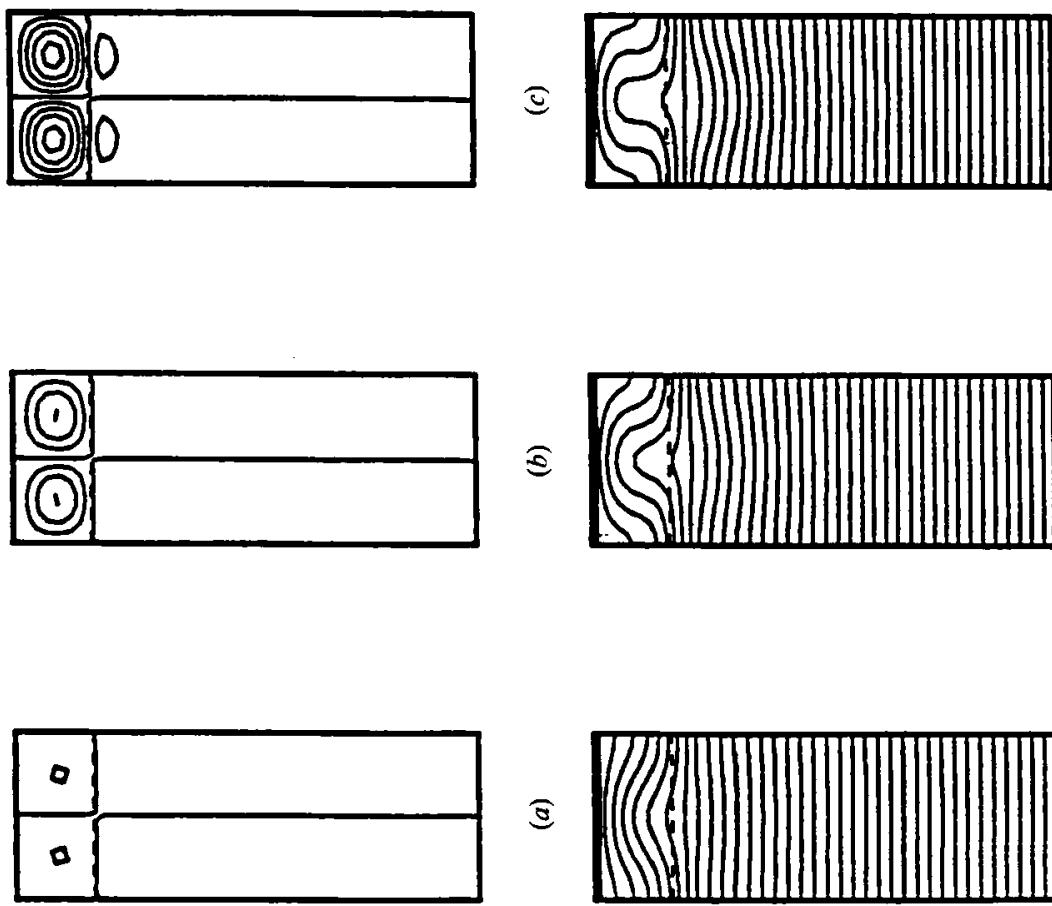

2
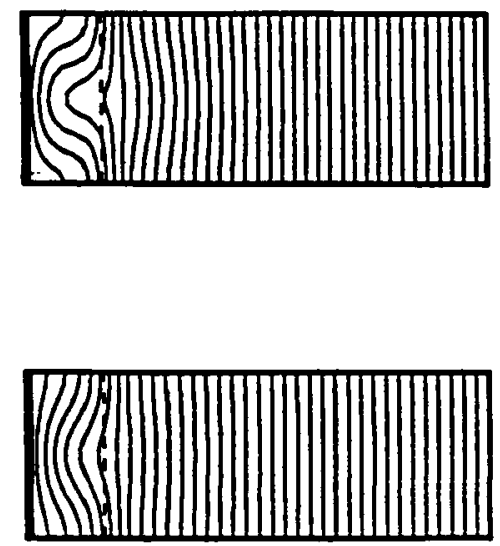


$\begin{array}{clccr}\hat{d} & R_{\mathrm{m}_{\mathrm{e}}} & R_{\mathrm{c}} & 20 R_{\mathrm{m}_{\mathrm{c}}} & 20 R_{\mathrm{c}} \\ 0.1 & 17.8 & 381 & 350 & 7620 \\ 0.2 & 4.85 & 1660 & 97 & 33200 \\ 0.5 & 0.132 & 1765 & 2.64 & 35300 \\ 1.0 & 0.0081 & 1732 & 0.16 & 34680\end{array}$

TABLE 5. Critical Rayleigh numbers at various depth ratios

(a)
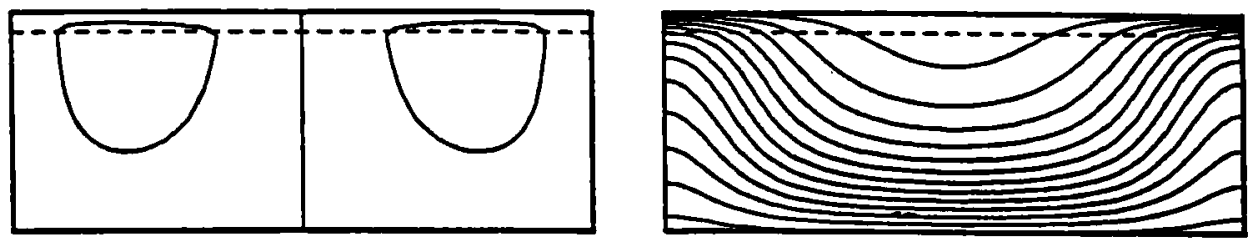

(b)
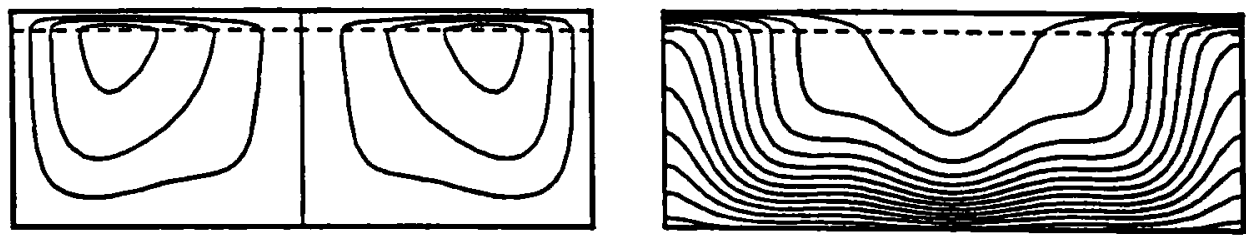

(c)
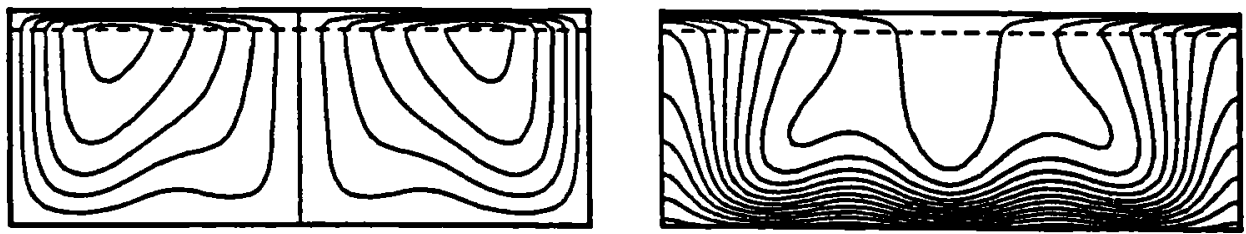

(d)
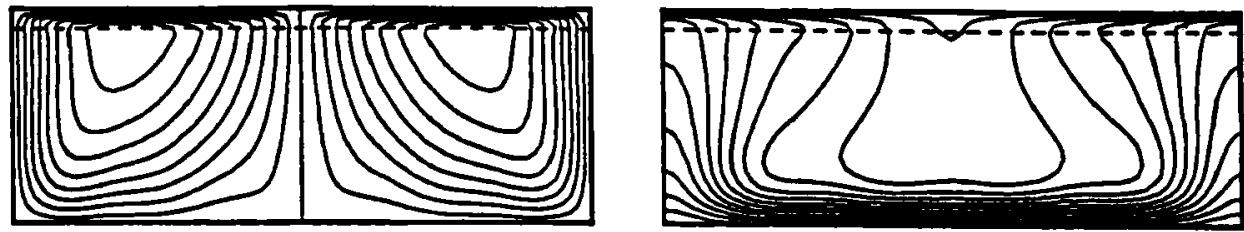

(e)
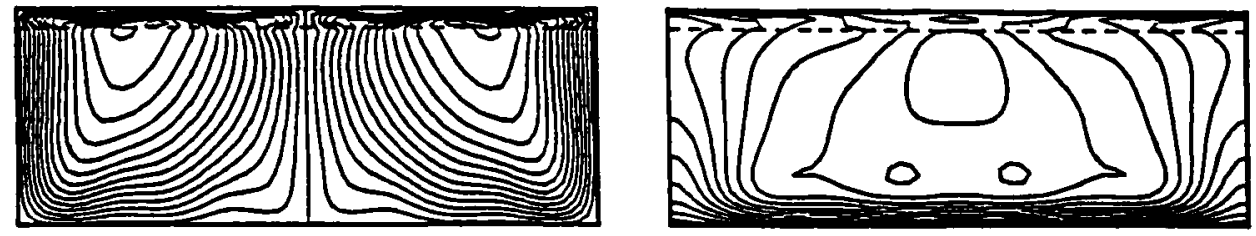

FIgURE 8 . As figure 4 but for $\hat{d}=0.1$ with $\Delta \psi=0.5$ and $\Delta T=0.1$. 


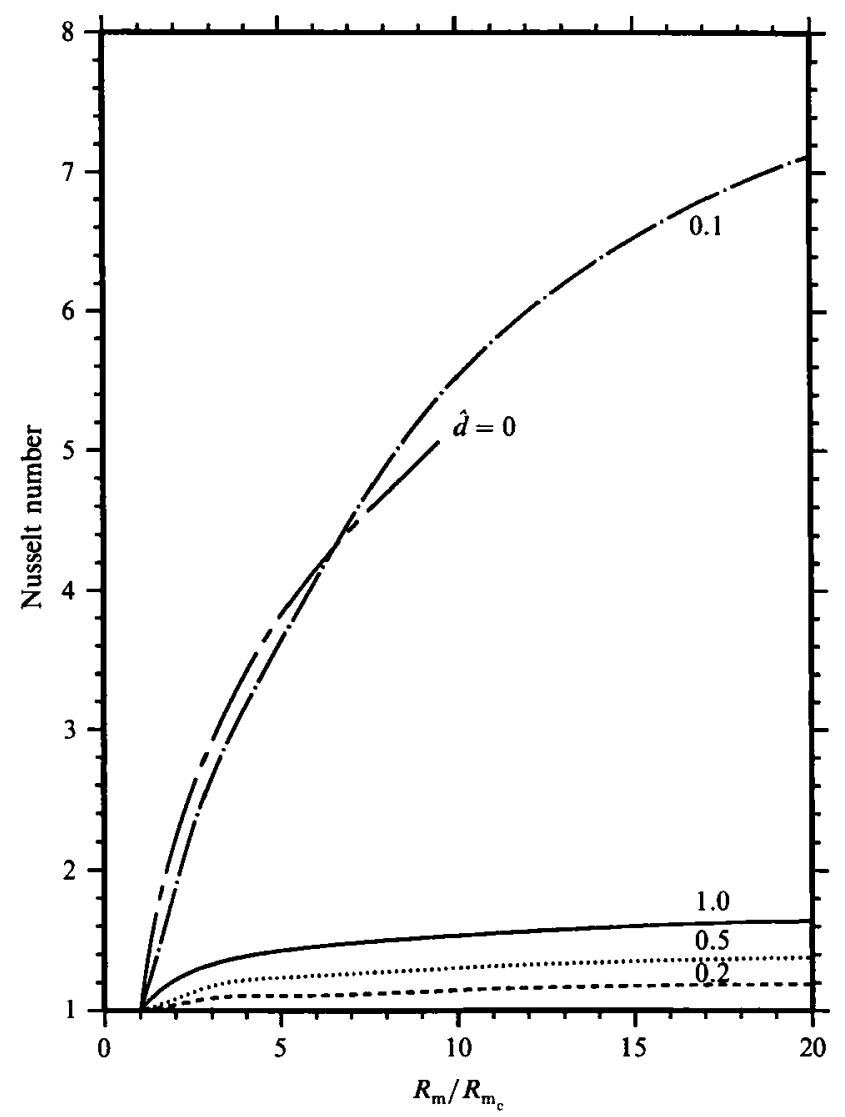

FigURE 9. Variation of Nusselt number with Rayleigh number for $\hat{d}=0,0.1,0.2,0.5$, and 1.0 .

quite closely, and the increase with $R_{\mathrm{m}}$ is quite appreciable, attaining a value of 7.13 at $R_{\mathrm{m}}=20 R_{\mathrm{m}_{\mathrm{f}}}$. As the depth ratio is increased beyond the critical value and the convection is fluid-layer dominated, the Nusselt number suffers a drastic decrease. This is because the porous layer, being non-convecting, serves as an insulating layer, as indicated in figure 7. Modest increase with layer depth is indicated. However, unless the fluid in the porous layer participates in the convection, the Nusselt number remains below 2 .

As a further check on our computational scheme, we have evaluated the Nusselt number for $\hat{d}=0.2$ at $R_{\mathrm{m}}=2 R_{\mathrm{m}_{\mathrm{c}}}$ and $3 R_{\mathrm{m}_{\mathrm{c}}}$ and $\hat{d}=0.1$ at $R_{\mathrm{m}}=1.5 R_{\mathrm{m}_{\mathrm{c}}}$ and $2 R_{\mathrm{m}_{\mathrm{c}}}$ using the same physical properties as those in the experiments we performed earlier (Chen \& Chen 1989). It is known that the heat transfer rate computed by assuming two-dimensional rolls in porous layers shows remarkable agreement with experiments in which the flow pattern is known to be three-dimensional. The computational results of Straus (1974) and Combarnous \& Bories (1975) compare favourably with the experimental results of Elder (1967), Combarnous \& LeFur (1969), and Buretta (1972). In the experiments of Combarnous \& LeFur, the three-dimensional convection cells were mapped by detailed measurements of the temperature distribution in the middle of the porous layer. Although no data were. presented about the convection pattern in the other two experiments, three-dimensional cells probably prevailed.

In the experiment by Chen \& Chen $(1989)$ with $\hat{d}=0.2$, the fluid used was a $60 \%$ 

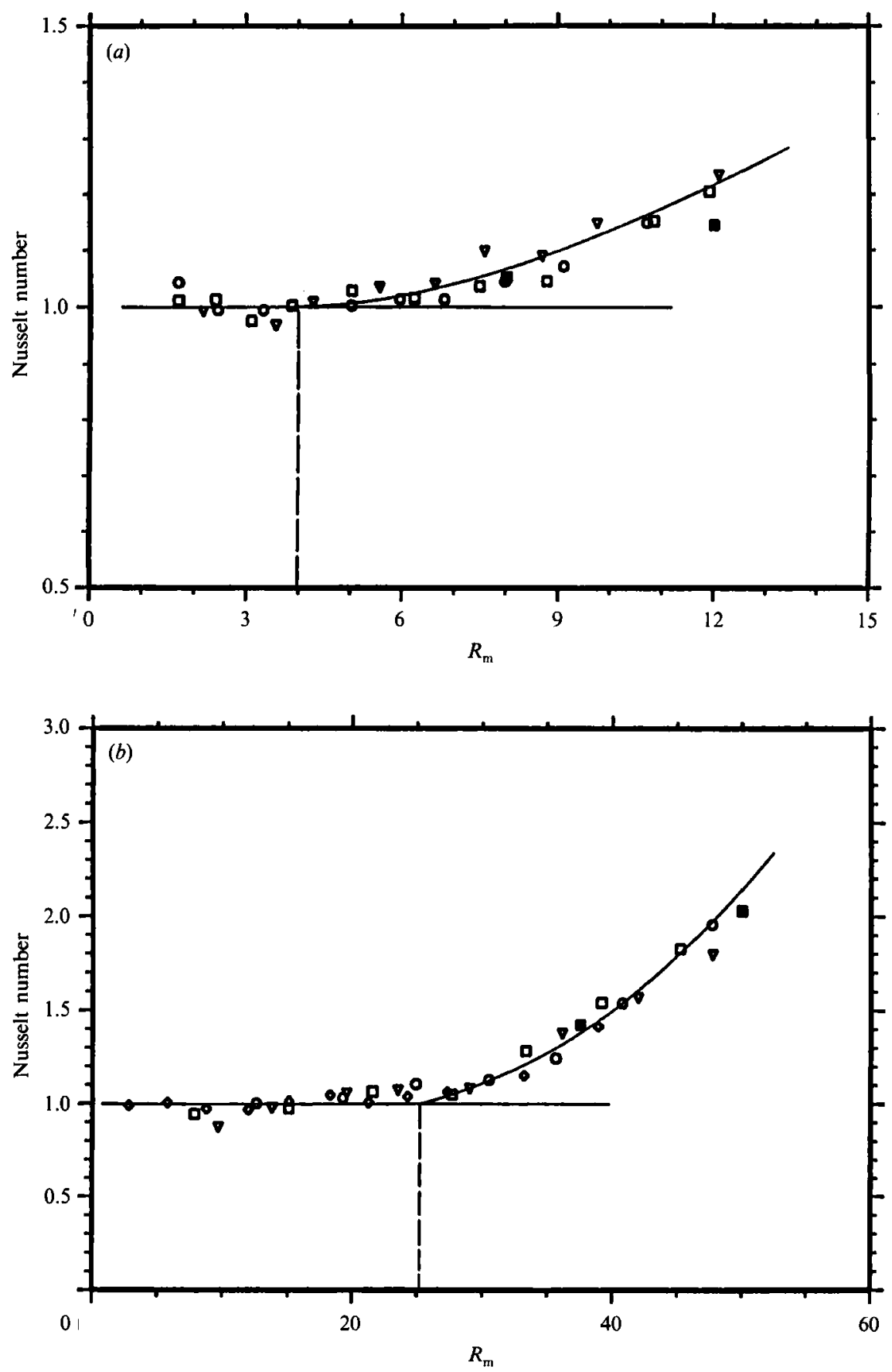

Fravre 10. Comparison of computed values of $N u$ with experimental results of Chen \& Chen (1989). The open symbols are experimental points and the filled squares are computed values. $(a) \hat{d}=0.2$, (b) $\hat{d}=\mathbf{0 . 1}$. 


\begin{tabular}{|c|c|c|c|c|}
\hline \multirow[b]{2}{*}{$\hat{d}$} & \multirow{3}{*}{$\begin{array}{c}R_{\mathrm{m}} / R_{\mathrm{m}_{\mathrm{c}}} \\
\left\{\begin{array}{l}2 \\
3\end{array}\right.\end{array}$} & \multicolumn{3}{|c|}{ Nusselt number } \\
\hline & & Experimental & Computational & Error \\
\hline 0.2 & & $\begin{array}{l}1.06 \\
1.21\end{array}$ & $\begin{array}{l}1.053 \\
1.145\end{array}$ & $\begin{array}{l}-0.8 \% \\
-5.7 \%\end{array}$ \\
\hline 0.1 & $\left\{\begin{array}{l}1.5 \\
2\end{array}\right.$ & $\begin{array}{l}1.38 \\
2.11\end{array}$ & $\begin{array}{l}1.425 \\
2.032\end{array}$ & $\begin{array}{l}+3.2 \% \\
-3.8 \%\end{array}$ \\
\hline
\end{tabular}

TABLE 6. Comparison between present computed results and the experimental results of Chen \& Chen (1989)

\begin{tabular}{cccc} 
& \multicolumn{3}{c}{$\operatorname{Pr}$} \\
\cline { 2 - 4 }$R_{\mathrm{m}} / R_{\mathrm{m}_{\mathrm{c}}}$ & 0.72 & 6.26 & 100.0 \\
1.5 & 1.134 & 1.134 & 1.134 \\
3.0 & 1.328 & 1.329 & 1.329 \\
5.0 & 1.426 & 1.423 & 1.422 \\
10.0 & 1.512 & 1.523 & 1.517 \\
20.0 & 1.595 & 1.620 & 1.594
\end{tabular}

TABLe 7. Nusselt number results for fluids with increasing Prandtl numbers at $\hat{d}=1.0$

glycerine-water solution with a Prandtl number of 100 , and when $\hat{d}=0.1$, the fluid was water. Comparisons are made in figures $10(a)$ and $10(b)$. In these figures, the experimental data points are unfilled symbols and the two computed values are represented by dark squares. It can be seen that agreement in both cases is very good. For a more detailed comparison, the values are listed in table 6 .

\subsubsection{Effect of Prandtl number}

To investigate the effect of Prandtl number on the characteristics of thermal convection, we have made calculations with $\operatorname{Pr}=0.72$ and 100 for $\hat{d}=1.0$, keeping all other physical properties the same as in the previous cases. Up to $R_{\mathrm{m}}=20 R_{\mathrm{m}_{\mathrm{c}}}$, no oscillations are encountered, similar to the case with water. The streamline patterns and the isotherm distributions are generally similar to those we have shown for water in figure 5. The Nusselt numbers at increasing Rayleigh numbers for $P r=0.72$, 6.26, and 100 are listed in table 7 . It is seen that the heat transfer rate is hardly affected by a more than 100-fold increase in the Prandtl number. This is because heat transfer in the porous layer is essentially by conduction.

\section{Conclusions}

The following conclusions may be drawn from the results of the present investigation :

(i) In superposed fluid and porous layers of equal thickness, the steady heat transfer rate calculated using the critical wavelength at onset of convection is either at or within $2 \%$ of the maximum value.

(ii) For the cases considered, $\hat{d}=0.1$ to 1.0 , convection remains steady up to 20 times the critical Rayleigh number.

(iii) Heat transfer rates for superposed layers with depth ratios less than the critical value increase much faster with Rayleigh numbers than those with depth 
ratios greater than the critical. This is because at low depth ratios, the convection is throughout the combined layer. At high depth ratios, convection is confined within the fluid layer and heat transfer is limited by heat conduction through the porous layer.

Financial support of this research provided by NASA Grant NAG-3-723 and the National Science Foundation Grant MSM-8702732 is gratefully acknowledged. We also wish to thank the National Science Foundation for providing computing time at the San Diego Supercomputer Center.

\section{REFERENCES}

Beavers, G. S. \& Joseph, D. D. 1967 Boundary conditions at a naturally permeable wall. J. Fluid Mech. 30, 197-207.

BeckermanN, C., Ramadhyani, S. \& Viskanta, R. 1986 Natural convection flow and heat transfer between a fluid layer and a porous layer inside a rectangular enclosure. In Natural Convection in Porous Layers (ed. V. Prasad \& N. A. Hussain), pp. 1-12. ASME.

Beckermann, C., Viskanta, R. \& RamadhyanI, S. 1988 Natural convection in vertical enclosures with fluid and porous layers. J. Fluid Mech. 186, 257-284.

BuEll, J. C. 1988 A mixed finite difference/Galerkin method for three-dimensional RayleighBénard convection. J. Comput. Phys. 75, 54-72.

BuRETta, R. J. 1972 Thermal convection in a fluid filled porous layer with uniform internal heat sources. Ph.D. dissertation, University of Minnesota, Minneapolis.

Caltagirone, J. P. 1975 Thermoconvection instabilities in a horizontal porous layer. J. Fluid Mech. 72, 269-287.

Chen, F. \& Chen, C. F. 1988 Onset of finger convection in a horizontal porous layer underlying a fluid layer. Trans. ASME C: J. Heat Transfer 110, 403-409.

Chen, F. \& Chen, C. F. 1989 Experimental investigation of convective instability in a superposed fluid and porous layer when heated from below. J. Fluid Mech. 207, 311-321.

Combarnous, M. A. \& Bories, S. A. 1975 Hydrothermal convection in saturated porous media. Adv. Hydrosci. 10, 231-307.

Combarnous, M. \& LeFur, B. 1969 Transfert de chaleur par convection naturelle dans une conche poreuse horizontale. C. R. Acad. Sci. Paris B 269, 1009-1012.

Curry, J H., Herring, J. R., Loncaric, J. \& OrszaG, S. A. 1984 Order and disorder in two-and three-dimensional Bénard convection. J. Fluid Mech. 147, 1-38.

ELDER, J. W. 1967 Steady free convection in a porous medium heated from below. J. Fluid Mech. $27,29-48$.

Erqun, S. 1952 Fluid flow through packed columns. Chem. Engng. Prog. 48, 89-94.

Georgiadis, J. G. \& Catton, I. 1986 Prandtl numbers effect on Bénard convection in porous media. Trans. ASME C: J. Heat Transfer 108, 284-290.

Goldhirsch, I., Peltz, R. B. \& Orszag, S. A. 1989 Numerical simulation of thermal convection in a two-dimensional box. J. Fluid Mech. 199, 1-28.

Krishnamurti, R. 1970 On the transition to turbulent convection. Part 2. The transition to timedependent flow. J. Flow Mech. 42, 309-320.

Lapwood, E. R. 1948 Convection of a fluid in a porous medium. Proc. Camb. Phil. Soc. 44, 508-521.

MacDonald, I. F., El-Sayed, M. S., Mow, K. \& Dullien, F. A. L. 1979 Flow through porous media - the Ergun equation revisited. Indust. Engng. Chem. Fundam. 18, 199-208.

McDonough, J. M. 1980 The Rayleigh-Bénard problem on a horizontally unbounded domain: determination of the wavenumber of convections. Ph.D. dissertation, University of California, Los Angeles.

MeYer-Spache, R. \& Keller, H. B. 1980 Computations of the axisymmetric flow between rotating cylinders. J. Comput. Phys. 35, 100-109. 
Neale, G. \& Nader, W. 1974 Practical significance of Brinkman extension of Darcy's law: coupled parallel flows within a channel and a boundary porous medium. Can. J. Chem. Engng $52,472-478$.

NiELd, D. A. 1977 Onset of convection in a fluid layer overlying a layer of a porous medium. J. Fluid Mech. 8, 513-522.

NiELD, D. A. \& JosePh, D. D. 1985 Effect of quadratic drag on convection in a saturated porous medium. Phys. Fluids 28, 995-997.

Nishimura, T., Takumi, T., Shiraishi, M., Kadamura, Y. \& Ozok, H. 1986 Numerical analysis of natural convection in a rectangular enclosure horizontally divided into fluid and porous regions. Intl J. Heat Mass Transfer 29, 889-898.

Peyret, R. \& Taylor, T. D. 1982 Computational Methods for Fluid Flow. Springer.

Poulikakos, D. 1986 Buoyancy-driven convection in a horizontal fluid layer extending over a porous substrate. Phys. Fluids 29, 3949-3957.

Pruetr, C. D. 1986 Numerical simulation of nonlinear waves in free shear layers. Ph.D. dissertation, University of Arizona, Tucson.

RABinowitz, P. H. 1968 Existence and nonuniqueness of rectangular solutions to the Bénard problem. Arch. Rat. Mech. Anal. 29, 32-57.

Roger, E. H. \& BEARD, D.W. 1969 A numerical study of wide-gap Taylor vortices. J. Comput. Phys. 4, 1-18.

Schubert, G. \& Straus, J. M. 1982 Transition in time-dependent thermal convection in fluidsaturated porous media. J. Fluid Mech. 121, 301-313.

Somerton, C. W. \& Catton, I. 1982 On the thermal instability of superposed porous and fluid layers. Trans. ASME C: J. Heat Transfer 104, 160-165.

Steen, P. H. \& AIdUn, C. K. 1988 Time-periodic convection in porous media: Transition mechanism. J. Fluid Mech. 196, 263-290.

Straus, J. M. 1974 Large amplitude convection in porous media. J. Fluid Mech. 64, 51-63.

Sun, W. J. 1973 Convection instability in superposed porous and fluid layers. Ph.D. dissertation, University of Minnesota, Minneapolis.

WARD, J. C. 1964 Turbulent flow in porous media. J. Hydraul. Div. ASCE 90, 1-13. 